RESEARCH REPORT

\title{
Mortality in children from influenza and respiratory syncytial virus
}

\author{
Douglas M Fleming, Rachel S Pannell, Kenneth W Cross
}

J Epidemiol Community Health 2005;59:586-590. doi: 10.1136/jech.2004.026450

See end of article for authors' affiliations .....................

Correspondence to: Dr D M Fleming, Birmingham Research Unit of the Royal College of General Practitioners, Lordswood House, 54 Lordswood Road, Harborne, Birmingham B179DB, UK; dfleming@ rcgpbhamresunit.nhs.uk

Accepted for publication 13 December 2004

\begin{abstract}
Study objective: To quantify mortality attributable to influenza and respiratory syncytial virus (RSV) infection in children.

Design and methods: Comparison of death rates (all cause and certified respiratory) in England over winters 1989/90 to 1999/00 during and outside influenza and RSV circulation periods. Virus active weeks were defined from clinical and virological surveillance data. Excess deaths associated with weeks of either influenza or RSV activity over virus non-active weeks were estimated in each winter for age groups 1-12 months, 1-4, 5-9, and 10-14 years. The estimate obtained was allotted to influenza and RSV in the proportion derived from independent separate calculations for each virus.

Main results: Average winter respiratory deaths attributed to influenza in children 1 month-14 years were 22 and to RSV 28; and all cause deaths to influenza 78 and to RSV 79. All cause RSV attributed deaths in infants 1-12 months exceeded those for influenza every year except 1989/90; the average RSV and influenza attributed death rates were 8.4 and 6.7 per 100000 population respectively. Corresponding rates for children $1-4$ years were 0.9 and 0.8 and for older children all rates were 0.2 or less, except for an influenza rate of 0.4 in children 10-14 years.

Conclusions: Influenza and RSV account for similar numbers of deaths in children. The impact varies by winter and between age groups and is considerably underestimated if analysis is restricted to respiratory certified deaths. Summing the impact over the 11 winters studied, compared with influenza RSV is associated with more deaths in infants less than 12 months, almost equal numbers in children 1-4 years, and fewer in older children. Improved information systems are needed to investigate paediatric deaths.
\end{abstract}

nfluenza is widely recognised as a substantial cause of winter excess mortality. ${ }^{1-6}$ Most of this excess, at least in recent years has been concentrated in persons over 65 years of age. ${ }^{6}$ In the influenza outbreak in late 2003 in the United States of America and in the UK there was considerable media concern about a small number of deaths in children. ${ }^{7}$ Respiratory syncytial virus (RSV) is also recognised as a cause of excess winter mortality particularly in young children and the elderly population. ${ }^{4-10}$ Quantification of the mortality attributable to each of these viruses is difficult because they often circulate at roughly the same time each winter; the clinical symptoms of persons infected are similar; and comparatively few cases are investigated virologically. ${ }^{4-10}$ Dual infections occur but it is unclear whether the clinical impact of dual pathogens is cumulative. ${ }^{11}$ This study examines national mortality data for England to estimate deaths attributable to influenza and RSV in children.

\section{METHODS}

National mortality data for England by date of death, age and underlying cause between 1989 and 2000 were provided by the Office of National Statistics. The study was based on deaths in winter from week 40 of one year to week 20 of the next (beginning of October to first week in May). All cause mortality data were examined as well as deaths classified by primary cause to respiratory diseases (chapter VIII, International Classification of Disease 9th revision). Deaths in children less than 4 weeks were not included because these are mainly attributable to problems associated with prematurity, congenital malformation, or obstetric complications. Deaths were grouped by age ( $1-12$ months, 1-4 years, 5-9 years, and 10-14 years) and assembled into weekly datasets aligned to the routine notification weeks of the
Communicable Disease Surveillance Centre of the Health Protection Agency (HPA).

We defined periods of influenza activity from clinical surveillance data on influenza-like illness linked to virological data using methods described elsewhere (table 1). ${ }^{12}$ RSV active weeks were those in which there were a minimum of 200 reports submitted to the HPA in children 0-1 year; (excepting week 52, which was deemed active if reports in weeks 51 and 01 exceeded 200). In the winters 1992/93 and 1997/98, influenza occurred comparatively late in the winter and there was a clearer separation of influenza and RSV than in other winters.

In each winter and for each age group we calculated the average weekly mortality rate in virus active and non-active weeks. The difference between these rates was multiplied by the number of virus active weeks to estimate the total virus attributable winter mortality rate. This was applied to the

Table 1 Influenza and RSV active periods by week number

\begin{tabular}{lll}
\hline Year & Influenza & RSV \\
\hline $1989 / 90$ & $46-3$ & $1-5$ \\
$1990 / 91$ & $51-10$ & $49-12$ \\
$1991 / 92$ & $51-9$ & $45-3$ \\
$1992 / 93$ & $8-16$ & $50-9$ \\
$1993 / 94$ & $42-51$ & $47-8$ \\
$1994 / 95$ & $1-14$ & $45-4$ \\
$1995 / 96$ & $45-3$ & $46-6$ \\
$1996 / 97$ & $49-9$ & $47-9$ \\
$1997 / 98$ & $6-14$ & $44-3$ \\
$1998 / 99$ & $51-6$ & $45-2$ \\
$1999 / 00$ & $50-5$ & $44-1$ \\
\hline
\end{tabular}


Table 2 Estimated virus attributable deaths in England over 11 winters 1989/90 to $1999 / 2000$ from all causes and from respiratory causes, by age

\begin{tabular}{lllllll}
\hline All causes & $\mathbf{1 - 1 2}$ months & $\mathbf{1 - 4}$ years & $\mathbf{5 - 9}$ years & $\mathbf{1 0 - 1 4}$ years & $\mathbf{1 5}$ +years & All ages \\
\hline Influenza & 422 & 233 & $\mathbf{7 5}$ & 128 & 183208 & 184067 \\
RSV & 534 & 238 & 27 & 66 & 161046 & 161912 \\
Total & 956 & 471 & 102 & 195 & 344255 & 345979 \\
Respiratory causes & & & & & & \\
Influenza & 129 & 80 & 4 & 25 & 83680 & 83916 \\
RSV & 185 & 94 & 5 & 24 & 69448 & 69756 \\
Total & 314 & 174 & 9 & 49 & 153127 & 153673 \\
\hline & & & & & & \\
\end{tabular}

national population to estimate the number of deaths attributable to each virus disease. The calculation was undertaken for influenza and RSV separately and then for the weeks in which either of the viruses was circulating (combined virus active period). As periods of activity for these viruses overlap, the sum of the individual estimates exceeded the combined. The estimates finally attributed to each virus were calculated from the combined estimate by allocation in the proportion of the individual virus estimates. (Thus, if the combined estimate was $\mathrm{C}$ and the estimates for individual viruses $\mathrm{I}$ and $\mathrm{R}$, the apportioned estimates were $\mathrm{CxI} /[\mathrm{I}+\mathrm{R}]$ and $\mathrm{CxR} /[\mathrm{I}+\mathrm{R}])$. For the presentation of selected results, we combined data from the 11 winters into eight 4 week winter periods. Although the study was essentially concerned with children we applied the methods to estimate attributable deaths in adults (15 years plus) as a reference frame to other material.

\section{RESULTS}

In the 11 winter periods studied there were 2857 deaths in children ( 1 month to 14 years) certificated to respiratory causes, $45.9 \%$ pneumonia, $1.4 \%$ influenza, $15.3 \%$ bronchitisbronchiolitis, $9.4 \%$ asthma, $2.9 \%$ to other alveolar pneumopathy (ICD code 516.8), 8.7\% to diseases of lung not elsewhere specified (ICD code 518.8), and $16.4 \%$ to the remaining specified respiratory diseases. Age specific estimates of all cause and respiratory deaths attributed to each virus were summed over the 11 winters and are given in table 2. The all age all cause total of 345979 deaths is equivalent to an average of 31000 per annum occurring during influenza and RSV active periods, more than $99 \%$ of which occurred in adults. The total of 153673 respiratory deaths is equivalent to an average of 14000 per year attributed to periods of activity of both viruses; 55\% attributed to influenza and $45 \%$ to RSV. In children, there were 546 respiratory deaths $(0.36 \%$ of all age total) and the relative proportions were $44 \%$ attributed to influenza and $56 \%$ RSV. Most (314) occurred in children less than 1 year where the RSV estimate exceeded the influenza estimate by $43 \%$. On average, influenza and RSV together accounted for 29 respiratory deaths per winter in infants (1-12 months), 16 in pre-school children (1-4) years, and five in school children (5-14 years): equivalent estimates for all cause deaths were 87 in infants, 43 in pre-school children, and 27 in school children.

Estimates of influenza and RSV attributable deaths in each four week period are presented as the total over 11 winters for children (1 month-4 years; fig lA) and adults over 15 years (fig 1B; note scale differences on the vertical axes). Estimates of attributable deaths in children (all cause and respiratory) were greatest in weeks $49-52$ and in adults in weeks $1-4$.

Estimates of respiratory deaths in influenza and RSV active periods are presented by winter as histograms (fig 2) for infants (1-12 months) and for children 1-4 years; (deaths in older children were very infrequent). For influenza, the estimates in infants $1-12$ months and in children $1-4$ years were highest in 1989/90 and substantially exceeded the equivalent RSV estimates; influenza activity was particularly high in November and December 1989. In all other years, estimates of RSV deaths exceeded the influenza equivalents. There was considerable winter variation in the estimates for both viruses. Combining the estimates for age groups 1-12 months and 1-4 years, there were more than 30 RSV attributed deaths in 1991/92, 1992/93, 1993/94, and 1999/00.

Age specific estimates of influenza and RSV attributed death rates (all causes and respiratory) averaged over the 11 winters studied are given in table 3, where the risk for infants 1-12 months relative to older children is emphasised. The nearest equivalent rates reported by Thompson et $a l^{10}$ are included in the table and considered in the discussion below. The average all cause attributable rates for infants aged 1-12 months were about nine times greater than the corresponding rates for children aged $1-4$ years; for the two older age groups rates were very small; a similar pattern is evident for attributed respiratory mortality rates.

\section{DISCUSSION}

The study found increased deaths in children during influenza and RSV active periods, particularly in infants 1-12 months. Averaged over the 11 winters studied, we estimate these two viruses account for an average of 50 certified respiratory deaths per year in children (1 month-14 years); 22 attributable to influenza and 28 to RSV: and of these, in the age group 1-12 months, 12 attributable to influenza and 17 to RSV. The equivalent estimates for all cause deaths are 157 ( 78 from influenza and 79 from RSV) and in the age group 1-12 months 87 ( 38 from influenza and 49 from RSV). The corresponding attributable rates highlight the difference between the impact in infants with that in older age groups.

All cause mortality includes deaths in children with serious comorbidity for whom acute respiratory infection may be the terminal event. We suspect comorbidities causing immune compromise are more prevalent in children 10-14 years than 5-9 years, as the older group have had longer opportunity to acquire serious illness bearing on their immune competence. The level of immunity conferred by infection with either RSV or influenza is limited because of antigenic drift. ${ }^{7}$ Nevertheless extended immunity after exposure may have been sufficient to explain the comparative protection of older people in the influenza pandemic of 1977, many of whom had been exposed to an influenza virus with at least some common antigenic features several years before. ${ }^{13}$

The all age (including adults) all cause average annual estimate of deaths attributable to influenza as derived here from the combined estimate for influenza and RSV in each winter separately, slightly exceeds (but is consistent with) the 12500 


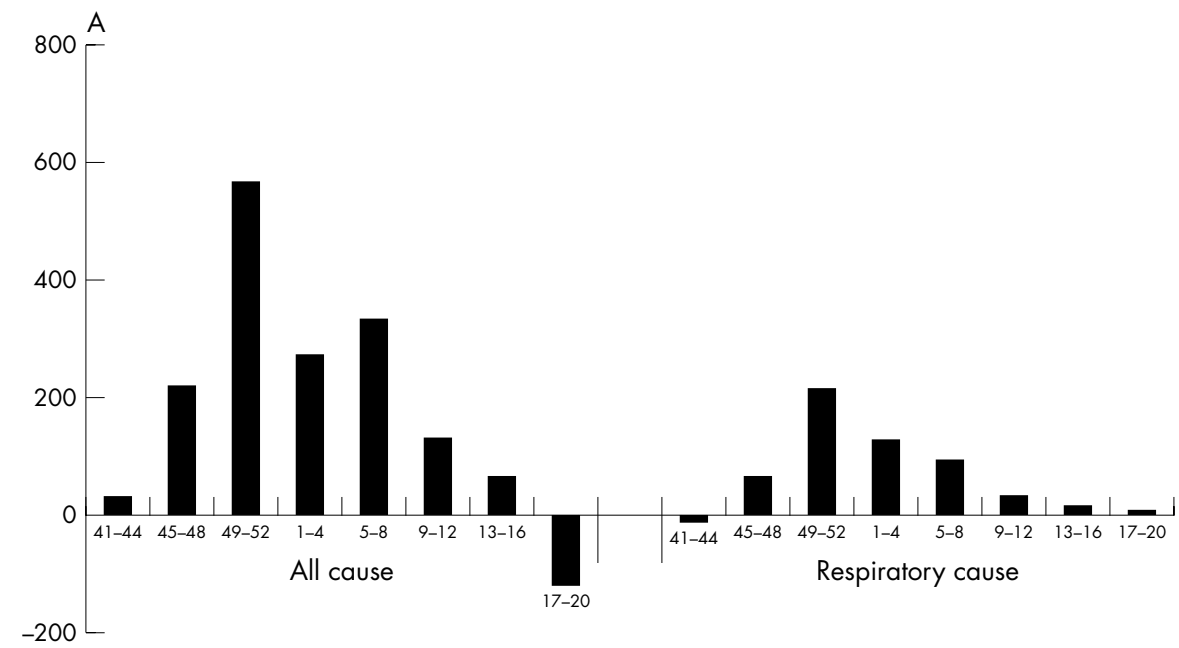

Figure 1 (A) Attributed deaths to influenza and RSV together, age 1 month to 4 years in four week periods (week 41 to 20) summed over 11 winters 1989/1990 to 1999/2000. (B) Attributed deaths to influenza and RSV together, age 15 years and over in four week periods (week 41 to 20 ) summed over 11 winters 1989/1990 to 1999/ 2000.
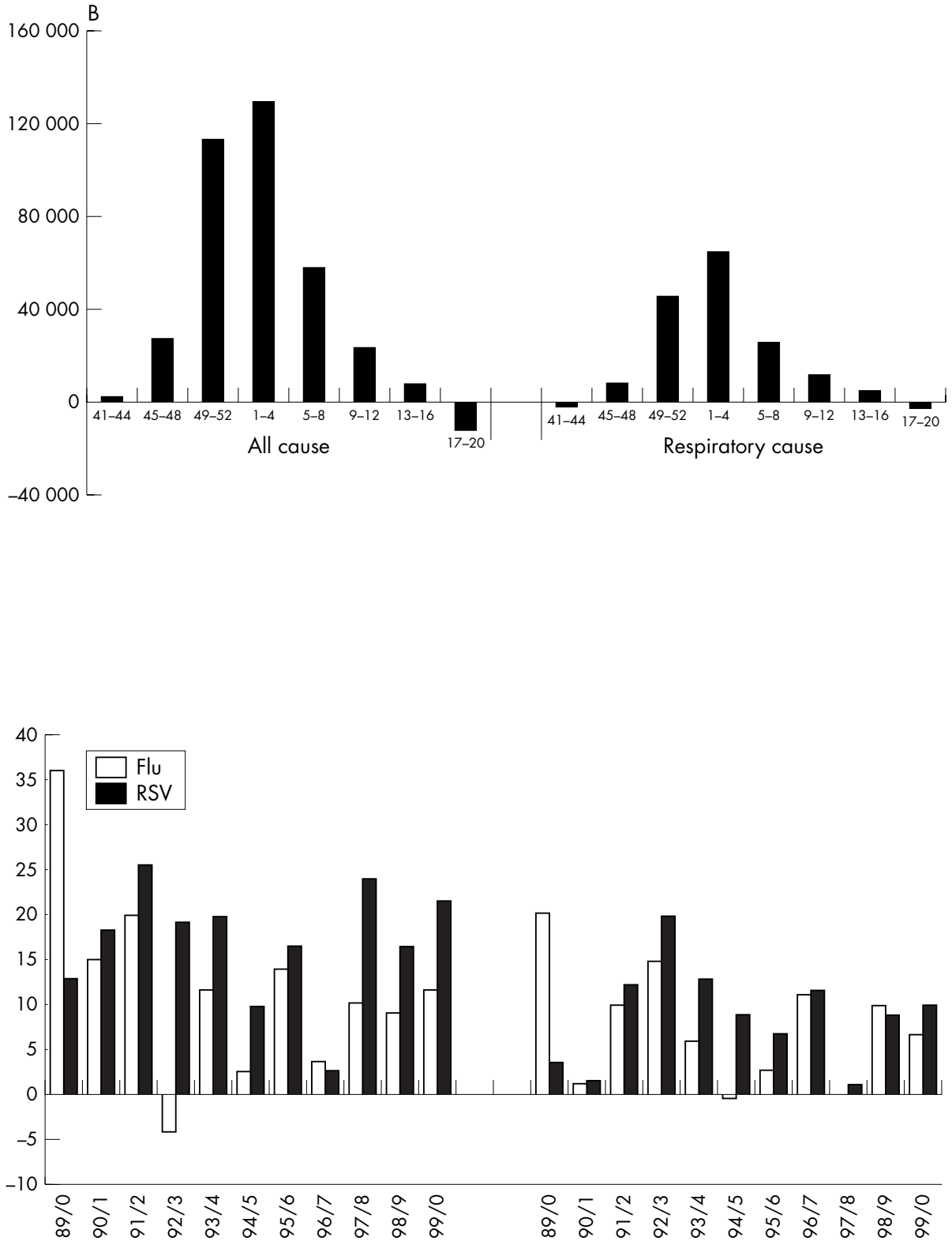

1-12 months

$1-4$ years

Figure 2 Influenza and RSV attributed respiratory deaths in each winter (1989/1990 to $1999 / 2000)$ for age bands 1-12 months and 1-4 years. 
Table 3 Average influenza and respiratory syncytial virus attributed winter mortality rate per 100000 by age group in winters $1989 / 90$ to $1999 / 00$

\begin{tabular}{|c|c|c|c|c|}
\hline All causes & 1-12 months & $1-4$ years & $5-9$ years & $10-14$ years \\
\hline Influenza & $6.7\left(2.2^{*}\right)$ & $0.8\left(1.1^{*}\right)$ & 0.2 & 0.4 \\
\hline RSV & $8.4\left(5.4^{*}\right)$ & $0.9\left(0.9^{*}\right)$ & 0.1 & 0.2 \\
\hline Total & 15.1 & 1.7 & 0.3 & 0.5 \\
\hline \multicolumn{5}{|c|}{ Respiratory causes } \\
\hline Influenza & $2.0\left(0.6^{*}\right)$ & $0.3\left(0.4^{*}\right)$ & 0.0 & 0.1 \\
\hline RSV & $2.9\left(5.3^{*}\right)$ & $0.3\left(0.2^{*}\right)$ & 0.0 & 0.1 \\
\hline Total & 5.0 & 0.6 & 0.0 & 0.2 \\
\hline
\end{tabular}

*Nearest equivalent reported by Thompson et al (see discussion).

calculated from average winter deaths in influenza active periods over the winters $1989 / 90$ to $1998 / 99$ using a common baseline of the average in all winters studied. ${ }^{6}$

The difference in the peak four week period for estimates of attributable deaths in infants (weeks 49-52) and the elderly (weeks 1-4) recognised in all cause and respiratory estimates, relates to the predominance of RSV infection in infants during weeks 49-52 that is seen consistently in RSV reports to the HPA. Deaths attributable to RSV in older age groups cannot be readily estimated from these data because the RSV active period in adults is less clear than it is in children. Simultaneous infection of all age groups may not be applicable to RSV, as it is to influenza. The combined all cause estimate for deaths attributable to influenza and RSV in adults is comparable with that published by Nicholson (this study average over 11 winters 31000 compared with Nicholson's average of 39000 over 15 winters $).{ }^{4}$ Individually we estimated about $53 \%$ of these were attributable to influenza and $47 \%$ to RSV, whereas Nicholson estimated RSV associated mortality substantially higher than influenza associated mortality. Excepting in children 0-4 years, the estimate of RSV attributed all cause mortality was less than that for influenza. Clinical incidence of acute bronchitis in children (0-4 years) as reported to the Weekly Returns Service is consistently highest in weeks $49-52,{ }^{14}$ which is the time when RSV reports to the HPA are maximal. In the elderly population the incidence of acute bronchitis is consistently highest in weeks one to four although it is uncertain which viruses are responsible.

Before comparing our results with those recently published from the USA by Thompson and colleagues, ${ }^{10}$ some differences in methods should be recognised (table 4): in particular, Thompson et al examined deaths in the age group less than 12 months and ourselves 1-11 months; analysed underlying respiratory and circulatory deaths and ourselves respiratory deaths; analysed deaths separately for influenza and pneumonia (although this category does not include acute bronchitis) and bronchiolitis. In the age group $1-4$ years our estimates for respiratory and for all cause mortality attributable to influenza and to RSV were similar to those reported by Thompson et al. In children 1-12 months our estimates for influenza were higher than the USA equivalents: for RSV our estimate was lower for respiratory deaths but higher for all cause mortality. To match the Thompson study period more closely, we re-examined our data excluding the winters 1989/90 and 1999/00, which in infants 112 months reduced the average influenza attributed mortality by about $20 \%$ (respiratory 1.6, all causes 5.9 ) but rates attributable to RSV scarcely differed. There is however no basis to presuppose that the incidence of virus infections should necessarily be similar in distant countries during similar periods. Although we found respiratory and all cause mortality attributable to RSV in this youngest age group exceeded that because of influenza, the difference was less than that reported by Thompson et al. However we noted with surprise their finding of no increase in the all cause mortality rate over that for respiratory and circulatory mortality.

Like Nicholson, ${ }^{4}$ we considered the difference between deaths in limited virus active/inactive periods as compared with a more general definition of seasonal differences. Thompson used methods that estimate attributable mortality over the whole year. As judged from the pattern of virus identifications reported to the HPA, the contribution of influenza and of RSV outside the winter period is likely to be extremely small and we believe it can safely be ignored. Neither RSV nor influenza is precisely confined to the active periods as described here, but by firstly considering the combined virus impact on mortality we restrict the likelihood of overestimation. However, other respiratory pathogens, and a winter increase of sudden infant deaths contribute to excess deaths during these periods. ${ }^{415}{ }^{16}$ Low temperatures also contribute to excess mortality but we could not find any reports of increased mortality in children directly attributable to cold weather. Circulatory deaths in children (combined with respiratory diseases in the estimate of Thompson et al) are rare and mostly caused by congenital anomalies.

The establishment of baseline levels is critical to these calculations. The limitations of cause specific certified

\begin{tabular}{lll} 
Table 4 & Comparison of study methods: Thompson et al and Fleming et al \\
\hline & Thompson et al, USA & Fleming et al, England \\
\hline Study period & Nine complete years (July to June) & Eleven winters (October to April) \\
Age groups & $1990 / 91-1998 / 99$ & $1989 / 90-1999 / 00$ \\
Methods & $<12$ months, 1-4 years, 5-49 years, & $1-12$ months, $1-4$ years, $5-9$ years, \\
& $50-64$ years, 65 years and over & $10-14$ years, 15 years and over \\
& Logistic regression using virus & Comparison of influenza and RSV active \\
Mortality end & positivity rates by week as a & and inactive weeks defined by clinical \\
points & marker of disease activity & and virological parameters \\
& Influenza/pneumonia deaths & Deaths from respiratory causes \\
& Underlying respiratory and & All cause mortality \\
& circulatory deaths & \\
& All cause mortality & \\
& &
\end{tabular}


What is known on this topic

RSV is major cause of acute bronchiolitis and is known to cause excess winter mortality in children especially in the first year of life. Influenza also causes occasional deaths in children.

\section{What this study adds}

This study quantifies excess mortality from RSV and influenza in children in England over the winters 1989/90 to 1999/ 00 .

mortality data and the difficulties of allocating mortality to specific respiratory viruses are widely recognised. In this study, only $1.4 \%$ of certified respiratory deaths were attributed to influenza. We defined the influenza active period from data on the clinical incidence of influenza-like illness and virus isolations; and the RSV active period from reports to HPA based on RSV tests. Thompson et al defined the influenza and RSV circulating periods from positivity rates among specimens tested, which are not available in England for the winters examined here. Both methods have their limitations. In recent years the specificity and sensitivity of virological tests have improved to the extent that more tests are undertaken. The impact of both illnesses is so variable between winters and even within age groups in an individual winter that it needs to be considered separately in each winter dataset. Notwithstanding the difficulties and the methodological differences between this study and that reported from the USA, both have produced similar estimates of combined influenza and RSV respiratory mortality in the critical 0-1 year age group, although from the English data the relative impact of influenza may be larger than that estimated from the USA dataset.

This study has been constrained by the limitations of using data linked from differing sources. As with the findings from studies in adults, it highlights the difference between estimates based on cause specific (respiratory) and all cause mortality. We believe the estimate of all cause mortality more accurately reflects the true impact of these virus conditions. Deaths in children are commonly investigated by postmortem examination. Our understanding of respiratory mortality and its impact could be greatly improved if results from antemortem and necropsy investigations could be linked with death certification details in a common dataset. Relevant information includes comorbidity, vaccination status, a list of all tests undertaken, and their results (positive or negative). Deaths in children are not so common for this to present excessive work.

The important conclusions from this study relate to the estimates of death attributed to influenza in young children. Even if not previously quantified precisely, RSV has been recognised as a cause of death in young children. These attributable mortality estimates in children are new and provide an important reference frame to determine policy in relation to influenza prevention and treatment in children. They are also valuable for interpreting the experience of the Fujian strain influenza outbreak at the end of the year 2003 and we look forward to having the data available whereby we might repeat this examination for that year.

\section{Key points}

- The all cause estimates of winter deaths in children attributable to influenza and RSV were three times those certified attributable to respiratory diseases.

- Estimates of the numbers of deaths in children attributable to influenza and RSV were broadly similar when averaged over several winters, although vary considerably between winters.

- Whether estimated separately by influenza or RSV; by certified respiratory or all causes; more than half the attributed deaths in children 1 month-14 years occurred in children 1-12 months.

\section{ACKNOWLEDGEMENTS}

Helpful comments on draft versions of the text were provided by $M$ Zambon of the HPA.

\section{Authors' affiliations}

D M Fleming, R S Pannell, K W Cross, Birmingham Research Unit of the Royal College of General Practitioners, Birmingham, UK

Funding: the Birmingham Research Unit is funded by the Department of Health who have given their permission to publish, although the opinions expressed in this report are the responsibility of the authors. No additional funding was provided for this study.

Competing interests: the authors have no conflicts of interest as far as this paper is concerned. D M Fleming has acted as an advisor and speaker on matters relating to influenza vaccination and treatment and to RSV epidemiology.

\section{REFERENCES}

1 Tillet HE, Smith JWG, Gooch CD. Excess deaths attributable to influenza in England and Wales; age at death and certified cause. Int J Epidemiol 1983; 12:344-52

2 Barker WH, Mullooly JP. A study of excess mortality during influenza epidemics in the United States 1968-76. Am J Epidemol 1982;115:479-80.

3 Glezen WP, Payne AA, Snyder DN, et al. Mortality and influenza. J Infect Dis 1982;146:313-20.

4 Nicholson KG. Impact of influenza and respiratory syncytial virus on mortality in England and Wales from January 1975 to December 1990. Epidemiol Infect 1996;116:51-63.

5 Simonsen L, Clarke MJ, Sconberger LB, et al. Pandemic versus epidemic influenza mortality: a pattern of changing age distribution. $J$ Infect Dis 1998;178:53-60.

6 Fleming DM. The contribution of influenza to combined acute respiratory infections, hospital admissions, and deaths in winter. Commun Dis Public Health 2000;3:32-8.

7 Treanor J. Influenza vaccine-outmaneuvering antigenic shift and driff N Engl J Med 2004;350:218-20.

8 Fleming DM, Cross KW. Respiratory syncytial virus or influenza? Lancet 1993:342:1507-10.

9 Crowcroft NS, Cutts F, Zambon MC. Respiratory syncytial virus: an underestimated cause of respiratory infection, with prospects for a vaccine. Commun Dis Public Health 1999;2:234-41.

10 Thompson WW, Shay DK, Weintraub E, et al. Mortality associated with Influenza and Respiratory Syncytial Virus in the United States. JAMA 2003;289:179-86

11 Zambon MC, Stockton JD, Clewley JP, et al. Contribution of influenza and respiratory syncytial virus to community cases of influenza-like illness: an observational study. Lancet 2001;358:1410-16.

12 Fleming DM, Zambon M, Bartelds AIM, et al. The duration and magnitude of influenza epidemics: A study of surveillance data from sentinel general practices in England, Wales and the Netherlands. Eur J Epidemiol 1999;15:467-73.

13 Potter CW. Chronicle of influenza pandemics. In: Nicholson KG, Webster RG, Hay AJ, eds. Textbook of influenza. Oxford: Blackwell Science, 1998:1-18.

14 Fleming DM. A perspective from general practice on selected influenza topics: near patient tests, influenza in pregnancy, influenza in children. In: Potter CW, ed. Influenza perspectives in medical virology 7. Amsterdam: Elsevier, 2002: 123-44.

15 Williams JV, Harris PA, Tollefson SJ, et al. Human metapneumovirus and lower respiratory tract disease in otherwise healthy infants and children. N Engl J Med 2004;350:443-50

16 Muller-Pebody B, Edmunds WJ, Zambon MC, et al. Contribution of RSV to bronchiolitis and pneumonia-associated hospitalizations in English children, April 1995-March 1998. Epidemiol Infect 2002;129:99-106. 